\title{
Hydrostatic pressure sensing using a polymer optical fibre Bragg gratings
}

\author{
Ian P. Johnson ${ }^{\mathrm{a}}$, David J. Webb ${ }^{\mathrm{a}}$, Kyriacos Kalli ${ }^{\mathrm{b}}$. \\ ${ }^{a}$ Photonics Research Group Aston University, Birmingham, B4 7ET, UK \\ ${ }^{\mathrm{b}}$ Nanophotonics Research Laboratory, Cyprus University of Technology, Limassol, Cyprus
}

\begin{abstract}
The sensitivity of a fibre Bragg grating (FBG) sensor fabricated in polymer optical fibre (POF) to hydrostatic pressure was investigated for the first time. In this initial investigative work a reflected Bragg response of a FBG fabricated in multimode microstructured POF (MMmPOF) was monitored, whilst the hydrostatic pressure was increased up to $10 \mathrm{MPa}$. Positive sensitivities were observed, meaning a positive wavelength shift to increasing pressure, as opposed to negative sensitivities monitored when using a FBG sensor fabricated in silica optical fibre. The FBG sensors fabricated in the MMmPOF gave fractional changes in wavelength and hence sensitivities of at least $64.05 \times 10^{-6} / \mathrm{MPa}$, which is some 25 times larger than the $-2.50 \times 10^{-6} / \mathrm{MPa}$ sensitivity of a FBG sensor fabricated in silica optical fibre that was measured in this work. Furthermore this work highlighted a decrease in sensitivity of the FBG sensor fabricated in the MMmPOF by some $50 \%$ by sealing the holes of the $\mathrm{mPOF}$ at the tip of the fibre with an adhesive. This offers the potential to tailor the response of the sensor to hydrostatic pressure.
\end{abstract}

Polymer optical fibre, fibre Bragg gratings, microstructured optical fibre, photonic crystal fibre, hydrostatic pressure sensitivity, PMMA.

\section{INTRODUCTION}

Fibre Bragg grating (FBG) fabrication in polymer optical fibre (POF) was first seen in 1999 [1], where step index (SI) POF was used based on poly (methyl methacrylate) (PMMA), and this remains the most widely used polymer for POF to date. Later, in 2005 Dobb et al [2] demonstrated the first fabrication of FBGs in microstructured POF (mPOF), which was manufactured purely from the PMMA polymer.

POFs have a number of advantages over their silica optical fibre counterparts; this includes their organic biocompatibility, which lends them to in-vivo biomedical applications [3]. Applications such as this also take advantage of the increased temperature sensitivity of FBGs fabricated in POF compared FBGs fabricated in silica optical fibre, where a $18 \mathrm{~nm}$ tuning range has been observed over a temperature range of $50^{\circ} \mathrm{C}$ [4]. Furthermore PMMA has a Young's modulus that is 25 times smaller than that of silica [5], and as a result recoverable strains of up to $13 \%$ have been demonstrated in PMMA based SI POF [6]. More recently, the interest in POF has increased due to the potential advantages they have in sensor applications, such as monitoring the strain in woven textiles of tapestries [7]. The motivation of this paper is to report any potential increased sensitivity of a FBG sensor fabricated in POF to hydrostatic pressure when compared to a FBG fabricated in silica optical fibre.

Increasing the hydrostatic pressure of the environment can result in a change in the Bragg wavelength of the response of a FBG through two different effects. Firstly, increasing the pressure will result in compression of the optical fibre and will thus decrease the period of the grating planes fabricated in the fibre core, leading to a negative Bragg wavelength shift. Secondly, the refractive index of the fibre increases as the fibre is compressed due to the increase in pressure, resulting in a positive Bragg wavelength shift. Therefore the sensitivity of the FBG to hydrostatic pressure can be given as:

$$
\frac{1}{\lambda} \frac{d \lambda}{d P}=-\frac{1}{E}\left((1-2 v)-\frac{n^{2}}{2}(1-2 v)\left(p_{11}+2 p_{12}\right)\right)
$$

Third Asia Pacific Optical Sensors Conference, edited by John Canning, Gangding Peng, Proc. of SPIE Vol. 8351, 835106 - (c) 2012 SPIE · CCC code: 0277-786X/12/\$18 · doi: 10.1117/12.914391 
Where $n$ is the Poisson's ratio and $\mathrm{p}_{11}$ and $\mathrm{p}_{12}$ are components of the relevant strain-optic tensor for an isotropic solid. With reference to equation (1), the first part in the parenthesis relates to the change in the period of the fabricated grating planes within the fibre core, whereas the second part relates to the refractive index change as a result of the strain optic effect. However, equation (1) lends itself to describe an isotropic solid, which is acceptable for silica optical fibre; conversely POF is not an isotropic material. During the drawing process of POF manufacture the molecular polymer chains tend to align along the fibre axis, and are therefore an example of a transverse isotropic material where the properties perpendicular to the fibre axis are not the same as the properties that are along the fibre axis. Therefore, the expression for the sensitivity to hydrostatic pressure of FBGs fabricated in POF are given as

$$
\frac{1}{\lambda} \frac{d \lambda}{d P}=-\left(\frac{1}{E_{T}}\left(1-2 v_{T}\right)-\frac{n^{2}}{2}\left(\left(\frac{1}{E_{p}}-\frac{v_{p}}{E_{p}}-\frac{v_{T}}{E_{T}}\right)\left(p_{11}+p_{12}\right)+\frac{p_{13}}{E_{T}}\left(1-2 v_{T}\right)\right)\right)
$$

where $E_{p}$ and $E_{T}$ are the elastic moduli parallel and perpendicular to the fibre axis, $v_{p}$ and $v_{T}$ are the relevant Poisson's ratios and $\mathrm{p}_{11}, \mathrm{p}_{12}$ and $\mathrm{p}_{13}$ are the components of the strain-optic tensor. A sensitivity of $-2.0 \times 10^{-6} / \mathrm{MPa}$ has previously been reported for a silica in-fibre Bragg grating exposed to hydrostatic pressures up to $70 \mathrm{MPa}[8]$.

\section{FIBRE BRAGG FABRICATION}

Multimode microstructured POF (MMmPOF) has been used in this first example of hydrostatic pressure sensitivity using POF. The MMmPOF that was provided by Kiriama Pty Ltd, was made from pure poly (methyl methacrylate) PMMA and was manufactured into fibre using the technique described by Barton et al [9], although the documented sleeving procedure was not implemented on the MMmPOF. The cleaved end face of the MMmPOF can be observed in Figure 1, the fibre had an outer diameter of $150 \mu \mathrm{m}$, as can be seen three rings of holes were used to provide index guiding within a $50 \mu \mathrm{m}$ solid core region.

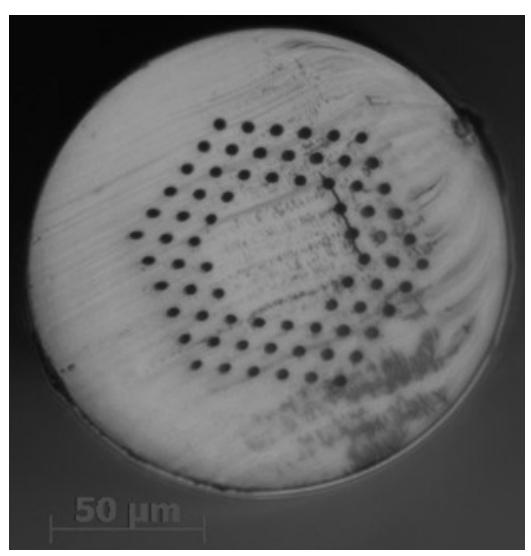

Figure 1 Microscope image of the end face of the MMmPOF, demonstrating a $150 \mu \mathrm{m}$ and a $50 \mu \mathrm{m}$ index guiding core.

Fabrication of a fibre Bragg grating (FBG) within the core region of the MMmPOF was undertaken using an ultra violet (UV), helium cadmium ( $\mathrm{HeCd}$ ) continuous wave (CW) laser, with an output wavelength of $325 \mathrm{~nm}$ and a specified power of $30 \mathrm{~mW}$. A relatively simple phase mask technique was used to fabricate the FBG when compared to fabrication setups for silica optical fibre. However, the essence of the setup was to ensure stability of the POF for fabrication times of up to one hour. It was ensured the MMmPOF was immobile during the FBG fabrication to prevent the fibre from sagging resulting in misalignment of the UV inscription laser beam; this was achieved by fixing the MMmPOF along a v-grooved plate, to prevent any movement using polyimide tape. The v-grooved plate was mounted horizontally onto a translation stage. The UV laser beam diameter $\left(1 / \mathrm{e}^{2}\right)$ was 
$1.2 \mathrm{~mm}$ which was also the length of the fabricated FBG in the MMmPOF, as no beam expansion or translation were utilised in the fabrication setup. Instead the UV beam was directed vertically down towards the MMmPOF and focussed onto the core of the fibre using a plano-convex cylindrical lens with a focal length of $10 \mathrm{~cm}$. Rested directly on top of the MMmPOF and supported by the v-groove plate was a phase mask with a surface relief pattern of period of $1057.2 \mathrm{~nm}$, the focussed UV laser beam passed through this phase mask and resulted in an UV interference pattern in the core of the MMmPOF with a period of $528.6 \mathrm{~nm}$. The alignment of the UV laser beam into the core of the MMmPOF was optimised by monitoring the scattering of the top surface of the POF.

FIBRE BRAGG FABRICATION

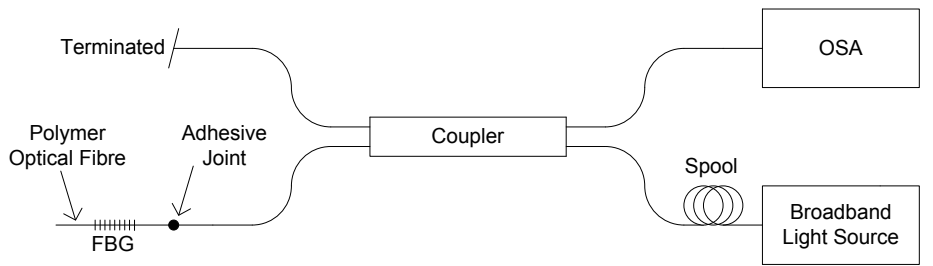

Figure 2 Experimental setup used to interrogate a reflected Bragg response of a FBG fabricated in MMmPOF

The experimental setup used to interrogate the reflected Bragg response can be observed in Figure 2. A $5 \mathrm{~mW}$ broadband ASE light source (Thorlabs ASE-FL7002-C4 5mW) was used to interrogate the Bragg response, this is an erbium source with an operating spectral range of $1.53-1.61 \mu \mathrm{m}$, and a typical spectral power density of $11 \mathrm{~dB} / \mathrm{nm}$ at $1540-1600 \mathrm{~nm}$. The broadband light source was passed through 20 metres of multimode $(50 / 125 \mu \mathrm{m})$ silica optical fibre that was wound around an $8 \mathrm{~cm}$ diameter spool to give an even modal distribution within the core of the multimode fibre. The interrogating light signal was then passed through a silica multimode $(50 / 125 \mu \mathrm{m}) 2 \times 2$ coupler with a $50: 50$ splitting ratio at $1550 \mathrm{~nm}$, and onto the fabricated FBG in the POF. The reflected Bragg response was captured via the coupler on an optical spectrum analyser (OSA) capable of receiving a multimode optical fibre (HP 70951B Spectral range 600-1700nm). The spare arm of the silica coupler was terminated with an angled connector (FC/APC), and index matching oil (RI=1.4587) was applied to the tip of the connector to reduce any Fresnel reflections from this coupler arm. The MMmPOF was permanently spliced to a silica multimode $(50 / 125 \mu \mathrm{m})$ pigtail, which was then connected to the arm of the coupler using FC/APC connectors. The polymer and silica fibres were spliced together using an optical adhesive (Loctite 3525) that was cured using a 60mW UV light source, the splicing technique is described in more detail in ref [10].

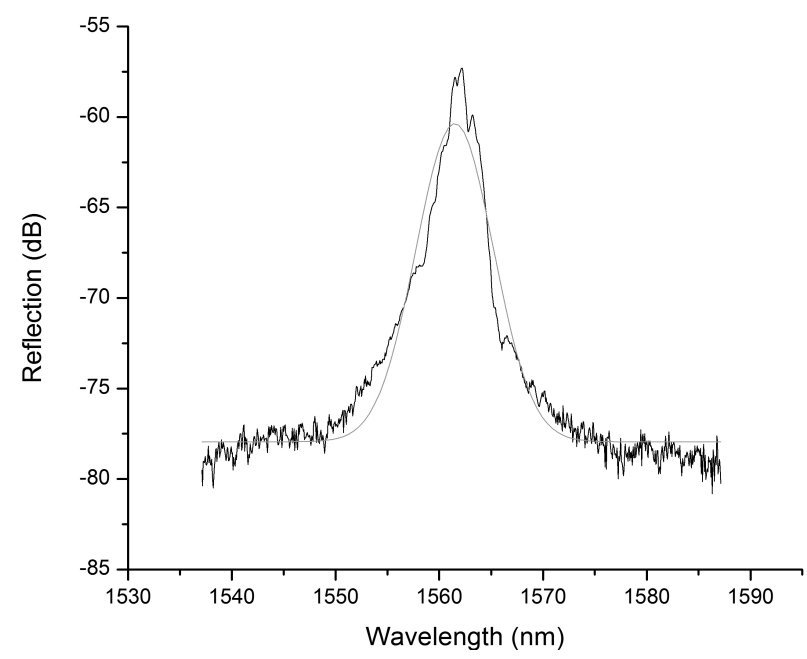

Figure 3 Reflected Bragg response of a FBG fabricated in MMmPOF measured using an OSA with a resolution bandwidth of $0.5 \mathrm{~nm}$, - Gaussian curve fit. 
Figure 3 shows the captured reflected Bragg response using the OSA, with a resolution bandwidth of $0.5 \mathrm{~nm}$ after a fabrication time of 50 minutes. Figure 3 also shows a Gaussian curve fit a Bragg wavelength of 1561.50nmm, a full wave half maximum (FWHM) bandwidth of $8.78 \mathrm{~nm}$ and a signal height of $17.58 \mathrm{~dB}$. Using a Gaussian fit gave a practical method of monitoring the centre wavelength on a broad reflected Bragg response.

Figure 4 demonstrates the experimental setup used to exert hydrostatic pressure on the POF up to $10 \mathrm{MPa}$ (100bar). The pressure chamber itself was a relatively basic setup; the fibre was suspended in the vertical steel tubing section of the chamber to aid the insertion and removal of the POF, whilst not damaging either the fibre or the adhesive splice between the polymer and silica optical fibres. Compressed air from a cylinder (200bar at $15^{\circ} \mathrm{C}$ ) was used to increase the hydrostatic pressure (monitored with a pressure gauge) within the chamber. The top of the chamber was sealed using a metal cap. Silica optical fibre was passed through the cap via a hole machined using a femtosecond laser, the silica fibre was stripped of the polymer protective buffer to prevent any slippage was permanently glued into the cap using Loctite 480 adhesive, thereby creating an air tight chamber.

Prior to the characterisation of the Bragg grating response to hydrostatic pressure, the pressure chamber was tested to $10 \mathrm{MPa}$ for 8 hours. During these initial tests a reversible $+2^{\circ} \mathrm{C}$ temperature increase of the chamber was observed when directly increasing the pressure from atmospheric pressure up to $10 \mathrm{MPa}$, also a $-2^{\circ} \mathrm{C}$ decrease in the temperature of the chamber was seen when releasing the pressure from $10 \mathrm{MPa}$ back to atmospheric pressure. The temperature of the chamber was monitored using a thermal couple attached to the outside of the vertical section of the chamber. After each observed temperature change, the temperature of the chamber returned to room temperature within 2 minutes.

When characterising the POF FBG sensitivity to hydrostatic pressure, the pressure was increased in $1 \mathrm{MPa}$ steps starting at $2 \mathrm{MPa}$ and stopping at $10 \mathrm{MPa}$. Two minutes were allowed from increasing the pressure and capturing the reflected Bragg response on the OSA so to allow any temperature dependent effects on the Bragg response to diminish.

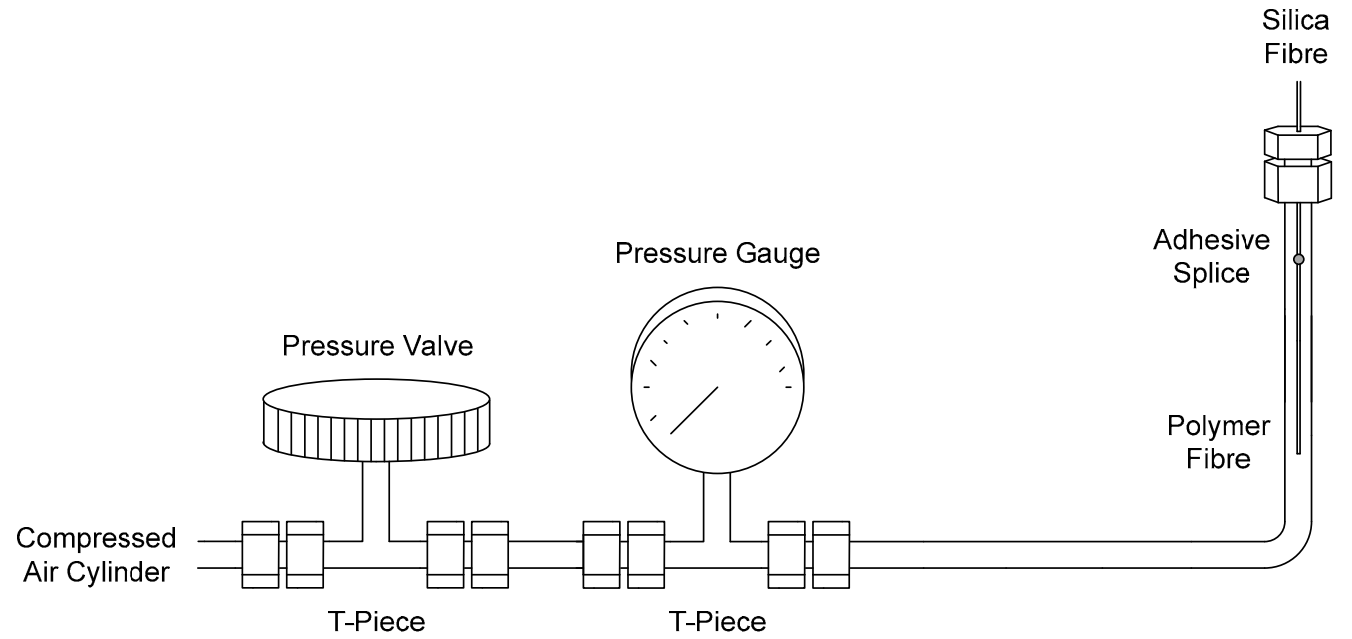

Figure 4 Experimental setup to test the hydrostatic pressure sensitivity of polymer optical fibre Bragg gratings

\section{RESULTS}

Initially the hydrostatic pressure sensitivity of a FBG sensor fabricated in single mode (9/125 $\mu \mathrm{m}) \mathrm{SMF} 28$ silica optical fibre was examined. The reflected Bragg response of this FBG is shown in Figure 5(a) with a Bragg wavelength of $1551.04 \mathrm{~nm}$ and a bandwidth (FWHM) of $0.22 \mathrm{~nm}$ was measured. The signal-to-noise ratio is $30 \mathrm{~dB}$. The reflected Bragg response was captured with a resolution bandwidth of $0.01 \mathrm{~nm}$ via a single mode $2 \times 2$ silica coupler, with a 50:50 splitting ratio at 1550nm. Figure 5(b) shows a total Bragg wavelength shift of - 
$31.01 \mathrm{pm}$ when increasing the hydrostatic pressure from $2 \mathrm{MPa}$ to $10 \mathrm{MPa}$, resulting in a sensitivity of $3.88 \mathrm{pm} / \mathrm{MPa}$ with a standard error of $0.04 \mathrm{pm}$. Furthermore, Figure 5(b) demonstrates that the dominant effect on the silica optical fibre with increasing hydrostatic pressure is the decrease of the period between the grating planes. Finally, dividing the sensitivity of $-3.88 \mathrm{pm} / \mathrm{MPa}$ by the Bragg wavelength $(1551.04 \mathrm{~nm})$ of the reflected Bragg response gives a fractional sensitivity of $-2.50 \times 10^{-6} / \mathrm{MPa}$, which is comparable to the sensitivity of $2.0 \times 10^{-6} / \mathrm{MPa}$ which has been previously reported[8].
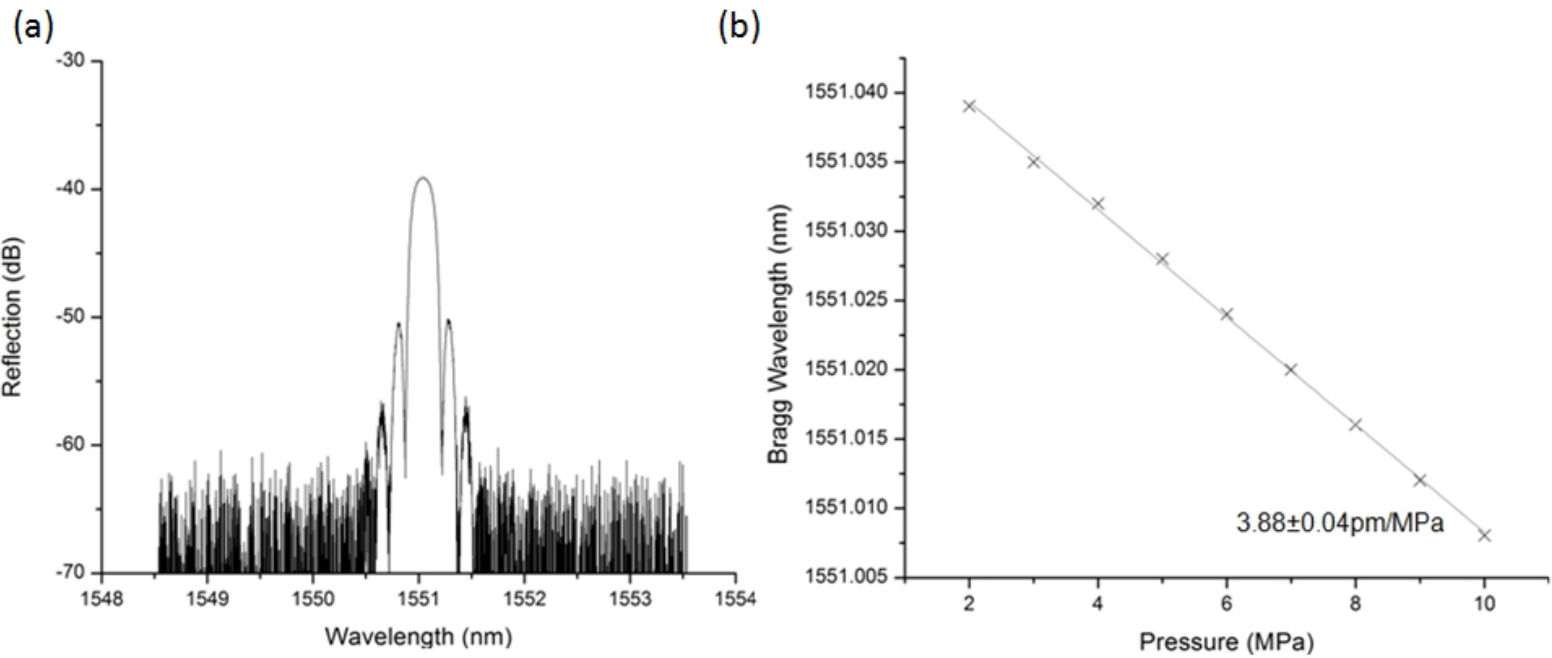

Figure 5(a) The reflected Bragg response of a FBG fabricated in SMF-28 silica optical fibre, (b) the linear response hydrostatic pressure of a FBG sensor fabricated in silica optical fibre (SMF-28).

The MMmPOF Bragg grating, whose wavelength response is shown in Figure 3, was then adhesive spliced to the silica optical fibre within the chamber and the same hydrostatic pressure conditions were applied as for the silica fibre Bragg grating. Results are presented in Figure 6(a \& b); Figure 6(a) shows the first Bragg wavelength shift due to an increase in the hydrostatic pressure up to $10 \mathrm{MPa}$, and Figure $6(\mathrm{~b})$ shows the sensitivity to the increase in pressure following the same procedure after the POF had been re-cleaved at both ends of the fibre and a new adhesive splice between the polymer and silica fibres was created. This was to examine the possibility of differing results as a result of a poor cleave or adhesive splice.

In both cases the sensitivity was positive with values of $0.10 \mathrm{~nm} / \mathrm{MPa}$ with a standard error of $9.38 \mathrm{pm}$ with the first test and $0.13 \mathrm{~nm} / \mathrm{MPa}$ with a standard error of $4.76 \mathrm{pm}$ for the second test. Both sets of data are in relative agreement with each other and would suggest with reference to equation (1) that the positive Bragg wavelength is a result of a refractive index increase being the dominating effect over a decrease in the period of the gratings planes of the fabricated FBG when the pressure within the chamber is increased. The fractional sensitivities to the hydrostatic pressure were also calculated to be $64.05 \times 10^{-6} / \mathrm{MPa}$ for test one and $83.25 \times 10^{-6} / \mathrm{MPa}$ for the second test. This indicates a sensitivity increase to hydrostatic pressure of at least 25 times compared with that of the FBG fabricated in silica optical fibre.

Following this initial sensitivity testing to hydrostatic pressure two further tests were completed where the holes of the mPOF at the tip of the fibre were sealed with adhesive (Loctite 480). Figure 6 (c \& d) show the sensitivity to an increase of hydrostatic pressure up to $10 \mathrm{MPa}$ on a FBG sensor fabricated on the same MMmPOF but now with the holes of the mPOF sealed at the tip of the fibre. The first test with a sealed fibre tip is shown in Figure 6 (c), which demonstrated a positive sensitivity of $0.07 \mathrm{~nm} / \mathrm{MPa}$ with a standard error of $2.23 \mathrm{pm}$. And Figure 6 (d) shows data collected from a second test with a sealed fibre tip, which gave a sensitivity of $0.09 \mathrm{~nm} / \mathrm{MPa}$ with a standard error of $3.27 \mathrm{pm}$. The fractional sensitivities were also calculated for each of these two tests, $44.83 \times 10^{-}$ ${ }^{6} / \mathrm{MPa}$ was calculated for test one with a sealed $\mathrm{mPOF}$ tip and $57.64 \times 10^{-6} / \mathrm{MPa}$ for the second test. We note that as a consequence of sealing the tip of the mPOF the sensitivity to hydrostatic pressure was reduced by nearly $50 \%$. This new set of data still gave a significant improvement on the sensitivity of hydrostatic pressure, which 
is at least18 times larger than that of a FBG sensor fabricated within the silica optical fibre. This change in sensitivity shows that the Bragg sensor in POF can have a tailored response to hydrostatic pressure.
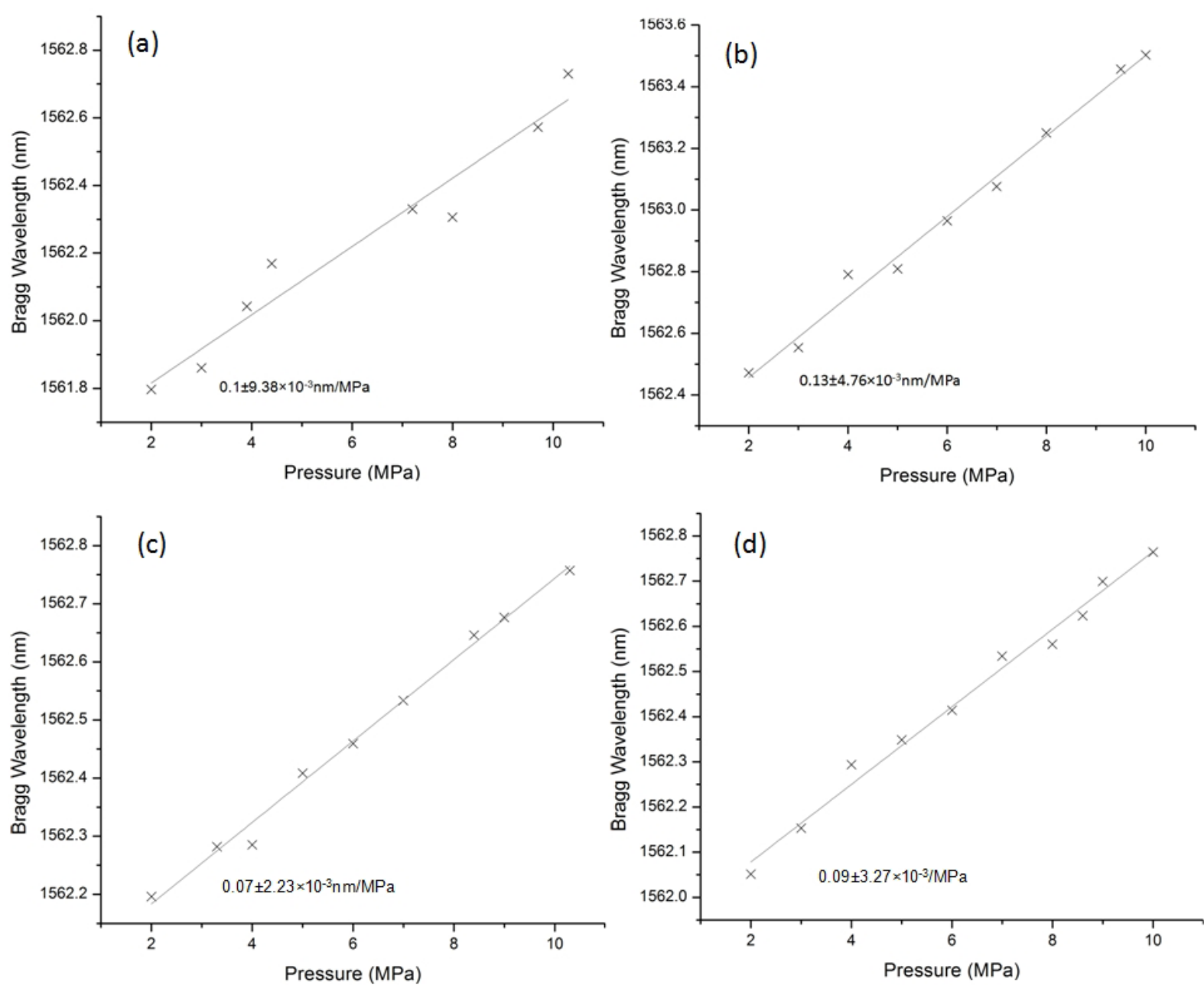

Figure 6(a) Test 1 of the hydrostatic pressure sensitivity of a FBG fabricated in MMmPOF, (b) test 2 of the hydrostatic pressure sensitivity of a FBG fabricated in MMmPOF, (c) first test of the hydrostatic pressure when the tip of the mPOF is sealed, (d) ) second test of the hydrostatic pressure when the tip of the mPOF is sealed.

\section{Conclusion}

To the best of our knowledge the sensitivity a FBG sensor fabricated in POF to hydrostatic pressure has been demonstrated for the first time. Fractional sensitivities as large as $83.25 \times 10^{-6} / \mathrm{MPa}$ were seen, which was some 33 times larger than $-2.5 \times 10^{-6} / \mathrm{MPa}$ measured when using a FBG sensor fabricated in SMF28 silica optical fibre. It was also discovered that as a result of sealing the holes of the MMmPOF at the tip of the fibre reduced the sensitivity by $50 \%$, suggesting a physical restriction on the increase of the period of the grating planes of the fabricated FBG. Nevertheless, this also offers the opportunity for a tailored hydrostatic pressure response.

When using equation (1) the positive sensitivity indicates as the pressure is increased the effect of the refractive index increasing due to the compression of the fibre dominates over the decrease of the period of the grating planes of the fabricated FBG. However, as already mentioned POF is not an isotropic material unlike silica optical fibre, rather POF is a transverse isotropic material due to the drawing process in the fibre manufacturing and should be described by equation (2). Alas independent measurements of the parameters needed for this equation to model the MMmPOF were not available. 
As may be seen from Figure 6 there is more noise in the data than that collected of the FBG sensor in silica optical fibre. This was a result of determining the Bragg wavelength of the broad reflected Bragg response.

\section{Acknowledgements}

The authors would like to acknowledge the European Commission for the funding of this work via the Framework 7 project PHOtonic For Optical Sensing (PHOSFOS). Furthermore the authors would like to thank Mr G Smith for the femtosecond laser machining of the cap to the pressure chamber to allow the insertion of optical fibre and also Mr C Mou for the fabrication of the FBG in the silica optical fibre.

\section{References}

[1] Z. Xiong, et al., "Highly tunable Bragg gratings in single-mode polymer optical fibers," IEEE Photonics Technology Letters, vol. 11, pp. 352-354, Mar 1999.

[2] H. Dobb, et al., "Continuous wave ultraviolet light-induced fiber Bragg gratings in few- and single-mode microstructured polymer optical fibers," Optics Letters, vol. 30, pp. 3296-3298, 2005.

[3] F. Baldini and A. G. Mignani, "Optical-fiber medical sensors," Mrs Bulletin, vol. 27, pp. 383387, May 2002.

[4] H. Y. Liu, et al., "Thermal tuning of polymer optical fiber Bragg gratings," IEEE Photonics Technology Letters, vol. 13, pp. 824-826, 2001.

[5] M. Silva-Lopez, et al., "Strain and temperature sensitivity of a single-mode polymer optical fiber," Optics Letters, vol. 30, pp. 3129-3131, Dec 2005.

[6] H. B. Liu, et al., "Strain and temperature sensor using a combination of polymer and silica fibre Bragg gratings," Optics Communications, vol. 219, pp. 139-142, Apr 2003.

[7] C. C. Ye, et al., "Applications of polymer optical fibre grating sensors to condition monitoring of textiles - art. no. 012020," in Sensors \& Their Applications Xv. vol. 178, A. T. Augousti and G. McConnell, Eds., ed Bristol: Iop Publishing Ltd, 2009, pp. 12020-12020.

[8] M. G. Xu, et al., "OPTICAL IN-FIBER GRATING HIGH-PRESSURE SENSOR," Electronics Letters, vol. 29, pp. 398-399, Feb 181993.

[9] G. Barton, et al., "Fabrication of microstructured polymer optical fibres," Optical Fiber Technology, vol. 10, pp. 325-335, Oct 2004.

[10] I. P. Johnson, et al., "Multiplexed FBG sensor recorded in multimode microstructured polymer optical fibre," Proc. SPIE 7714, 77140D (2010); doi:10.1117/12.854410, Brussels. 